\section{Slosh simulation in a computer model of canine syringomyelia}

\author{
Clare Rusbridge ${ }^{1,2}$, Serge Cirovic ${ }^{1}$ \\ 1 Univeristy of Surrey, Guidlford, United Kingdom \\ 2 Fitzpatrick Referrals, Godalming, United Kingdom
}

\section{OBJECTIVES}

The exact pathogenesis of syringomyelia is unknown. Epidural venous distention during Valsalva manoeuvres (raised intrathoracic pressure when expiring against a closed glottis e.g. coughing) causing impulsive movement of fluid ("slosh") within the syrinx is a proposed cause of syrinx dissection into spinal cord parenchyma and craniocaudal propagation of the cavity. We sought to test the "slosh" hypothesis by epidural excitation of CSF pulse in a computer model of canine syringomyelia.

\section{METHODS}

Our previously developed canine syringomyelia computer model was modified to include an epidural pressure pulse. Simulations were run for: a cord with small syringes at $\mathrm{C} 2$ and the cervicothoracic junction (locations previously shown prone to syrinx formation); a healthy cord; and cord with a large syrinx.

\section{RESULTS}

If small syringes are present, there are peaks of stress at those locations. For a single cervical syrinx, stress is highest at the caudal end of the syrinx, whereas for a cervicothoracic syrinx stress is highest at the cranial end of the syrinx. When there are both small cervical and cervicothoracic syringes, the stress peaks remain similar to each separately. However, there are no pressure peaks with a large syrinx. The stress is slightly elevated in the caudal cord up to the caudal end of the syrinx.

\section{STATEMENT (CONCLUSIONS)}

The findings supported the "slosh" hypothesis suggesting that small cervical syringes might propagate caudally and thoracic syringes cranially. However, when the syrinx is large there is less focal stress which may explain why a syrinx can expand rapidly but then remain unchanged in shape over years.

\section{Thoracolumbar} intervertebral foraminal and far lateral disc extrusions in dogs: clinical signs, MRI findings and shortterm outcome

\section{Sara Silva ${ }^{1}$, Roberto José-López ${ }^{1}$, Steven De Decker², Josep Brocal ${ }^{3}$, Julien Guevar", Cristian de la Fuente ${ }^{5}$, Alexane Durand ${ }^{4}$, Franck Forterre ${ }^{4}$, Rodrigo Gutierrez-Quintana ${ }^{1}$}

1 School of Veterinary Medicine, College of Medical Veterinary and Life Sciences, University of Glasgow, Glasgow, United Kingdom

2 Queen Mother Hospital for Animals, The Royal Veterinary College, University of London, London, United Kingdom

3 Wear Referrals Veterinary Hospital, Bradbury, United Kingdom

4 Vetsuisse Faculty, University of Bern, Bern, Switzerland

5 Hospital Clinic Veterinari, Universitat Autònoma de Barcelona, Barcelona, Spain

\section{OBJECTIVES}

The aim of this study was to describe the clinical signs and MRI findings, as well as to assess short-term outcome after medical or surgical treatment in dogs diagnosed with thoracolumbar intervertebral foraminal and far lateral disc extrusions.

\section{METHODS}

The medical records of five referral institutions were retrospectively searched between 2009 and 2019 for dogs diagnosed with thoracolumbar (T3-L6) intervertebral foraminal and/or far lateral disc extrusions. Twenty-one dogs were identified and included.

\section{RESULTS}

Cocker Spaniels and Dachshunds were the most commonly affected breeds. Median age at presentation was 5 years, weight $12 \mathrm{Kg}$ and time from onset to presentation was 17.5 days. Most frequent clinical findings were pain on spinal palpation and/or hip extension (86\%) and pelvic limb lameness (71\%). Caudal lumbar intervertebral disc extrusions (L5-L6 or L6-L7) were present in $86 \%$ of the cases. All cases in which contrast was administered had nerve root or perilesional contrast enhancement on MRI. Surgical decompression was performed in $66 \%$ of the cases and $33 \%$ were treated with strict rest and a combination of gabapentin and NSAIDs or steroids. Improvement was observed in all surgically treated cases and the three medically treated in which follow-up was available. Median time of follow-up was 28 days. 


\section{Oral presentations}

\section{STATEMENT (CONCLUSIONS)}

To the authors' knowledge, this is the first case series describing the clinical signs, MRI findings and outcome of dogs diagnosed with this uncommon presentation of a common condition.

\section{Suspected 3-hydroxy- 3-methylglutaric aciduria (HMGA) in domestic shorthaired cats}

\section{Natalie West ${ }^{1}$, Kaspar Matiasek ${ }^{2}$, Clare Rusbridge ${ }^{1,3}$}

\footnotetext{
Fitzpatrick referrals, Surrey, United Kingdom University of Munich, Munich, Germany

3 University of Surrey, Surrey, United Kingdom
}

\section{OBJECTIVES}

3-hydroxy-3-methylglutaric aciduria (HMGA) is a metabolic disorder due to deficiency of 3 -hydroxy-3methylglutaryl-coenzyme A (HMG CoA) lyase, an enzyme involved in processing leucine and manufacturing ketones. HMGA is a rare $(<1 / 100,000)$ autosomal recessive condition in humans, associated with metabolic crisis, stunted growth, psychomotor retardation, epileptic seizures and spasticity. We aim to describe the presentation of suspected HMGA in 4 cats.

\section{METHODS}

We describe the neurological and diagnostic findings in 4 stray kittens ( 2 consecutive litters) presented to a charity.

\section{RESULTS}

The only male kitten was presented at $\sim 8$ weeks old with a gait abnormity which progressed over a month until euthanasia. His litter sister had a slower course with initial signs at 12 weeks; ultimately euthanatized at 5 months old. Two further female cats were presented at $\sim 8$ weeks old. All had an unusual gait with marked increased extensor muscle tone of the thoracic limbs giving a pronounced "goose stepping action" with spinocerebellar ataxia and intention tremor. They frequently fell, increased extensor tone and spasticity made rising difficult. Organic acid urinalysis from the first female revealed a HMGA of $45 \mathrm{mmol} / \mathrm{mol}$ creatinine (normal 0-2). Management with L-Carnitine at $100 \mathrm{mg} / \mathrm{kg} /$ day did not result in clinical improvement. Post mortem samples were obtained from 3 of 4 cats; preliminary results showed axonal degeneration of olivopontocerebellar and spinocerebellar circuits. Genetic analysis is in process.

\section{STATEMENT (CONCLUSIONS)}

We describe a spontaneously occurring metabolic disorder characterised by marked increased extensor tone. Understanding the pathogenesis may inform on analogous conditions in all species 